\title{
Spectral variability versus radial-velocity changes in WR 1
}

\author{
Andrzej Niedzielski \\ Toruñ Centre for Astronomy, Nicolas Copernicus University, \\ ul. Gagarina 11, 87-100 Toruñ, Poland
}

Abstract. We discuss the possibility that WR 1 (HD 4004) is a binary system.

\section{Introduction}

WR 1 (HD 4004) is one of several Wolf-Rayet stars which, inspite of their apparently single character, do show some variations in emission-line profiles. These variations do not have a regular periodic nature. They may be connected with binarity, or some, yet unknown, process inside the WR envelope.

In the case of WR 1, several aspects of profile variations have been presented in Niedzielski $(1995,1996,1999)$. Here we try to check out the other possibility, that WR 1 is a binary star, as was suggested before (Hackwell, Gehrz \& Smith 1974; Helfand 1980; Lamontagne 1983).

\section{Method}

The best method to prove binarity is to find a radial-velocity solution. In the case of WR stars, where the spectra are dominated by strong and broad emission lines and absorption features are absent, this is a big problem.

To observe radial-velocity changes in the optical spectra of WR 1 , we obtained spectra during five nights at a resolution of $1 \AA /$ pixel, covering both He II $\lambda 5411$ and the interstellar absorption features $\mathrm{NaI} \lambda \lambda 5890.0,5895.9$. The Na I lines were used for fixing possible wavelengths shifts due to the spectrograph flexure.

We have measured both the position of the center of the He II line (by fitting two Gaussian profiles: one to this line and second to its electron scattering red wing), and the central wavelength of the line. The later is not connected to the radial-velocity, but rather to the profile variations.

It is clear from our measurements that the variations of the position of the central intensity of the He II emission line are almost one order of magnitude larger than the measured radial-velocities as determined from the position of the line center. The wavelength of central intensity may vary up to $300 \mathrm{~km} \mathrm{~s}^{-1}$. These variations are not strictly periodic, but the formal sinus-wave with amplitude of $156 \mathrm{~km} \mathrm{~s}^{-1}$ and period of some 5.5 days fits them reasonably well. The radial-velocity variations, within the five days of observations, are larger than the observational errors. Careful reduction of the radial-velocities, and their nightly averages, results in a smooth run of radial velocities. A sinus-wave with 
an amplitude of $18 \mathrm{~km} \mathrm{~s}^{-1}$ and a period of some 6 days fits these data quite well. The significance of this fit is, unfortunately, marginal. The amplitude of $18 \mathrm{~km} \mathrm{~s}^{-1}$ is very small compared to the used spectral resolution. We estimate the uncertainty of the radial-velocity determination in one measurement as $\sim 20-30 \mathrm{~km} \mathrm{~s}^{-1}$. Most of our data were gathered within three consecutive nights, preceeded and followed by two nights with two and one measurement, respectively.

\section{Results and conclusions}

Within five nights of spectroscopic monitoring of WR 1 in the range $5200-5900 \AA$ with $1 \AA /$ pix resolution (58 spectra), we found that the radial-velocity of He II $\lambda 5411$ has a mean value of $13 \mathrm{~km} \mathrm{~s}^{-1}$. The mean values for the different nights vary by no more than $18 \mathrm{~km} \mathrm{~s}^{-1}$. However, the scatter within one night may be as large as $50 \mathrm{~km} \mathrm{~s}^{-1}$, which may be the result of the limited resolution in use. Although the amplitude of the radial-velocity variations is in agreement with the value of the semi-amplitude as derived from $\mathrm{He}$ II emission lines by Lamontagne (1984), we can not confirm that result, since our data do not fit the published ephemeris. At the same time, the profile variations, for unknown reasons, are ten times larger than the variations of the central intensity wavelength of the same line. These variations perturb the observed profile from the center down to some $50 \%$ of it's central intensity. Such strong variations of the observed profile may be reflected in the measured radial-velocities. The similarity of periods seen in these variations supports this conclusion. Also possible variations of the electron-scattering red-wing of this line may contribute to observed wavelength of He II $\lambda 5411$.

We suggest therefore that from our observations it appears unlikely that WR 1 is a binary.

Acknowledgments. This paper was supported by the KBN grant 2P03D 00512 through N. Copernicus University.

\section{References}

Hackwell, J.A., Gehrz, R.D., Smith, J.R. 1974, ApJ 192, 383

Helfand, D.J. 1980, PASP 92, 691

Lamontagne, R. 1983, Ph.D. Thesis, Univ. Montreal

Niedzielski, A. 1995 in: K.A. van der Hucht \& P.M. Wiliams (eds.), Wolf-Rayet Stars: Binaries, Colliding Winds, Evolution, Proc IAU Symp. No.163 (Dordrecht: Kluwer), p. 52

Niedzielski, A. 1996 in: in: J.-M. Vreux, A. Detal, D. Fraipont-Caro, E. Gosset \& G. Rauw (eds.), Wolf-Rayet Stars in the Framework of Stellar Evolution, Proc. $33^{r d}$ Liège Int. Astroph. Coll. (Liège: Univ. of Liège), p. 277

Niedzielski, A. 1999, Acta Astron. in press 